\title{
A Multiobjective, Maximal Conditional Covering Location Problem Applied to the Relocation of Hierarchical Emergency Response Facilities
}

\author{
Nicholas R. Paul ${ }^{\mathrm{a}}$, Brian J. Lunday ${ }^{\mathrm{a}}$, Sarah G. Nurre ${ }^{\mathrm{b}}$ \\ ${ }^{a}$ Department of Operational Sciences, Air Force Institute of Technology, \\ Wright Patterson AFB, OH 45433, United States \\ ${ }^{b}$ Department of Industrial Engineering, University of Arkansas, \\ Fayetteville, AR 72701, United States
}

\begin{abstract}
In this research we analyze the effectiveness of the current and optimal locations of a set of existing regional assets maintained by the Department of Defense to respond to large-scale emergencies. These assets have been incrementally resourced, established, sited over the past 20 years without regard to the entire enterprise and, due to fiscal and political costs, modifications to the current structure must yield significant gains to garner approval. We formulate a multiobjective hierarchical extension of the maximal covering location problem that seeks to maximize coverage of the population within a rapid response window while minimizing modifications to the existing structure. Additionally, we prevent facilities from covering nodes located within close proximity using a modified conditional covering problem (CCP) constraint; this constraint accounts for the large impact radius that can occur in a worst-case scenario. To solve our multiobjective problem, we develop a set of non-inferior solutions using the $\varepsilon$-constraint method. These non-inferior solutions explicitly represent the trade-off between maximizing coverage and minimizing cost, and they offer a decision maker a set of Pareto optimal decisions to consider for implementation. Applying our model and methodology to the current set of assets, we demonstrate that, in the absence of resource constraints, we can improve coverage by
\end{abstract}

Email address: brian.lunday@afit.edu (Brian J. Lunday)

Preprint submitted to Journal of Omega

December 4, 2015

(C) 2016. This manuscript version is made available under the Elsevier user license http://www.elsevier.com/open-access/userlicense/1.0/ 
more than 15\%, approximately 49 million people. Furthermore, with only 23 unit relocations (less than a $30 \%$ modification of the entire structure) coverage can exceed 98\%, an improvement of an additional 45 million people covered. Finally, we demonstrate additional advantages of implementing the modified CCP constraint.

Keywords: maximal covering location problem, large scale emergency, conditional covering problem, multiobjective optimization, $\varepsilon$-constraint method

\section{Introduction}

The motivation for this study is to examine the current and optimal locations for the Chemical Biological Radiological Nuclear (CBRN) Response Enterprise (CRE) in the Continental United States to better mitigate the effects of a CBRN incident on the population. The CRE consists of units from both the National Guard and active components of the Department of Defense that support local authorities with the (i) analysis of CBRN impacts, (ii) development of mitigation measures, (iii) initial medical stabilization and triage, (iv) search and extraction, and (v) decontamination. Locating these units in close proximity to potential targets reduces the response time and therefore improves the response capability. It is important, however, to maintain a minimum safe distance (i.e., a stand-off distance) between the CRE and priority demands to prevent a CBRN attack from neutralizing response capability. Moreover, because these units have already been sited, minimizing the cost of modifications is also desired.

The National Guard component of the CRE, on which our research focuses exclusively, is comprised of three echelons: (i) Weapons of Mass Destruction Civil Support Teams (WMD-CSTs), (ii) CBRN Enhanced Response Force Packages (CERFPs), and (iii) Homeland Response Forces (HRFs). WMD-CSTs constitute the first response of the CRE. Their primary missions are to assist first responders with the identification of CBRN effects and advise local and 
state officials on the impacts and protective measures [1]. There are currently 52 WMD-CSTs located in the Continental United States with at least one in each state and one in Washington D.C.. Moreover, Florida, California, and New York each have two WMD-CSTs due to their relatively larger population sizes. Each WMD-CST consists of approximately 22 personnel [2]. The CERFP is the second level of response, and their unit capabilities include casualty search and extraction, emergency medical services (EMS), and decontamination [2]. The CERFP is specifically designed to perform each of these tasks in a CBRN contaminated environment. There are currently 15 CERFPs located across the Continental United States with at least one per Federal Emergency Management Agency (FEMA) region, and each is comprised of approximately 186 personnel. HRFs comprise the third echelon of response. Each HRF consists of approximately 570 personnel and provides the same capability as a CERFP, but also adds a brigade-size command and control headquarters and a battalion-size security component. A HRF is designed to manage up to five WMD-CSTs and three CERFPs [3]. There are currently ten HRFs, one aligned with each FEMA region.

Facility location theory has been extensively applied to optimize the establishment of emergency management services such as fire departments and hospitals. More recently, the field has grown to examine location decisions that support response to large-scale emergencies (LSEs). LSEs are unique in their low frequency and catastrophic effect on a population, and they require different modeling considerations than classical facility location models [4]. Most of the previous LSE research focused either on optimal locations for the pre-positioning of medical supplies or on post-event establishment of relief aid distribution centers. There is limited work, however, that focuses specifically on recommending improvements to an existing LSE response structure, and much of it is focused on temporary asset relocation strategies. (See [5] and 6] for works that represent foundational and recent advancements in this area.) This is an important consideration, given the CRE is a significant LSE response structure in place within the United States, and a series of myopic location decisions successively 
implemented over increments to the size of the CRE has likely yielded suboptimal unit dispositions. Thus, an examination of these existing structures utilizing LSE facility location theory can assess their effectiveness. Furthermore, we can recommend modifications to balance the trade-off between improved service and the cost to attain it. These costs include both a monetary cost (i.e., the amount of money needed to relocate an organization, build new facilities, etc.) and political costs (e.g., removing an existing capability from an area to improve service in a different area). Within the scope of this research, we minimize the number of modifications to existing unit locations as a substitute for both the monetary and political costs, but we refer the reader to a preceding work by Paul 7 for a more detailed analysis of selected financial costs for altering the locations of CRE unit locations.

LSE models commonly employ concepts from covering models that have been widely applied to siting emergency management facilities such as hospitals and fire stations (e.g. see [8, 9, 10, 11]). In these models a demand is considered covered if a facility is located within a minimum service distance of a demand. Common covering models include the location set covering problem (LSCP) and the maximal covering location problem (MCLP). The LSCP was first applied to emergency management facility location by Toregas et al. [10, and it seeks to minimize the weighted cost of siting facilities such that every demand is covered by at least one facility within a maximum service distance. The maximal covering location problem (MCLP), first introduced by Church and Revelle [8], seeks to maximize coverage of a population within a defined coverage radius given a set number of facilities. The MCLP is commonly applied to public sector problems when resource limitations prevent covering all demands [12] and, unlike the LSCP, accounts for the size and location of demands [4.

Similar to many facility location problems, LSE problems often have multiple objectives [13, 14, 15]. The most common of these problems balance maximizing some measure of capability with minimizing cost [16, 17, 18, 19, 20, 21. Other notable bi-objective approaches include balancing coverage with distance [22, 23], coverage with equity [24, 25], or coverage efficiency with coverage equity 
[26. For a thorough review of multiobjective facility location problems, we refer the reader to a robust survey conducted by Farahani et al. [27] that applies taxonomies for both the number and types of objectives considered within the respective works, a notable subset of which address EMS location problems. We note that EMS location problems are one type of LSE problem under the aforementioned assumptions of low frequency and catastrophic effect for events; however, not all EMS location problems are examined as an LSE problem, and LSE problems are not restricted to EMS systems.

Among recent works published since the survey conducted by Farahani et al. [27, we note the following contributions as evidence of the growth in this field. Zhang and Jian [28] examined the location of EMS stations to balance the maximization of area coverage with the minimization of costs due to station construction, vehicle operating costs, and transportation costs. Within this work, the authors considered stochastic demand distributions and adopted a robust optimization approach, examining tradeoffs in the respective objectives. Rath and Gutjahr 29] modeled the location of humanitarian aid warehouses and routing of supplies to meet demands for relief goods, balancing three objectives: minimizing short-term costs consisting of depot construction, vehicle procurement, and vehicle operation; minimizing medium-term costs consisting of transportation and warehousing expenses; and maximizing the total demand satisfied by the design. Recent work by Khodaparasti et al. [26] explored the Pareto frontier of efficient solutions for designing an EMS system via location decisions that seek to minimize the expected response times and minimize the total number of uncovered demand zones. Adopting a greater emphasis on often underserved population in rural areas, Chanta et al. 24] identified locations for vehicular ambulances to balance four objectives: maximizing the weighted demand covered; minimizing the number of uncovered demand zones; minimizing the number of uncovered rural demand zones; and minimizing the maximum distance between uncovered demand zones and their respective closest open ambulance stations.

Ehrgott and Gandibleaux [30] provide a useful survey of methods to solve 
multiobjective facility location problems. Although there are many techniques available to solve multiobjective problems, solution generation methods are often preferred when access to a decision maker is limited [31. These methods involve generating all (or a sufficient sampling) of the non-inferior solutions. A solution is said to be non-inferior (or nondominated) for a multiobjective problem if an improvement in one objective can be accomplished only by degrading at least one other objective [32]. The set of non-inferior solutions allows a decision maker to consider a range of alternatives that explicitly define the trade-offs between competing objectives [16]. The decision maker then applies judgment to determine the desired balance between competing objectives [16].

The two most widely used generation methods are the weighted sum method and scalarization techniques, the latter of which include the $\varepsilon$-constraint method, the hybrid method, and the elastic constraint method [32. The weighting method involves parametric scaling of each objective in accordance with its relative importance and evaluating the corresponding objective function for a set of weights 33 . Among the various scalarization techniques, the $\varepsilon$-constraint method is more often utilized within recent multiobjective facilitation location literature (e.g., see [21, 24, 26, 29, 34, 35]). The $\varepsilon$-constraint method involves iteratively optimizing one objective after converting the remaining objectives into constraints, for which the right-hand side (RHS) is parametrically changed to identify a set of non-inferior solutions [35]. Chankong et al. [36] showed that the $\varepsilon$-constraint method is guaranteed to find the entire non-inferior set for a general multiobjective problem given a proper increment of the $\varepsilon$-value. It has also been demonstrated that an optimal solution to the $\varepsilon$-constraint method is guaranteed to be non-inferior if it is feasible and all the constraints representing objectives are binding 31. The $\varepsilon$-constraint method is often preferred over the weighting method in integer programming formulations because the non-convex feasible region prevents the weighting method from finding all non-inferior solutions. For this reason, the use of the weighted sum method in the literature is less frequent (e.g., see [22, 28]), and several authors have sought to improve upon it via the use of fuzzy set optimization [17, or evolutionary algorithms [19, 37, 
optimizing a ratio of two objectives [26, or a combination of the weighted sum and $\varepsilon$-constraint methods [20]. Herein, we embrace the use of the $\varepsilon$-constraint method for its simplicity and, for our formulation in which one of the objectives assumes only discrete values, its ease of identifying appropriate values for $\varepsilon$ to identify all non-inferior solutions.

The majority of research that focused exclusively on LSE facility location has occurred in the last ten years. Jia et al. 4, 38, developed models focused on proactively locating medical stockpiles and reactively siting distribution centers that would distribute medical supplies after a large-scale emergency. Murali et al. [39] developed a generalized capacitated MCLP, wherein coverage was determined using a decreasing step function of the distance, to optimize the distribution of medical supplies during a catastrophic bio-terror attack. In a related contribution, Peker and Kara [40] examined the $p$-hub maximal covering location problem, for which the authors considered gradual decay functions for partial demand coverage by facilities in lieu of binary coverage, as well as multiple facility contributions to determine the coverage of each demand. Sheu [41] developed a model focused on optimizing emergency logistic support during an LSE with imperfect information. Abounacer et al. 42, utilized the $\varepsilon$-constraint method to solve a multiobjective location-transportation problem for disaster response. Their model incorporated three objectives: minimize the travel distance of products, minimize the assets needed to open and operate distribution centers, and minimize the uncovered demand.

Several LSE models have also adopted elements of the conditional covering problem $(\mathrm{CCP})$ to model the large-impact radius of a LSE. The CCP was first introduced by Moon and Chaudhry 43 , with the same objective as the LSCP - to establish the minimum number of facilities to cover all demands - but the authors added an additional constraint requiring each sited facility to be covered by another facility. The original CCP motivation was intended to model facility interdependency. Lunday et al. 44] developed a modified version of the CCP wherein the set of demands and the set of potential facility locations were the same, and a facility cannot cover a co-located demand. The development of 
this modified version was intended to model the siting of Weapons of Mass Destruction Civil Support Teams (WMD-CSTs) that exist within the National Guard to support local authorities in the event of a WMD incident. Since a WMD incident could render a response unit ineffective, Lunday et al. 44] proposed preventing facilities from covering co-located demand. Huang et al. [45] developed a similar model for an LSE using a $p$-center objective that seeks to minimize the maximize distance between demands and facilities.

We note that our research invokes the term 'hierarchical' in a manner that differs slightly from its use in previous location theory literature. Beginning as early as 1979 in work by Narula and Ogbu [46] on location-allocation problems, hierarchical facilities traditionally refer to an interrelated structure of facility classifications wherein only the lowest level of facilities services demands, the next higher level of facilities supports the lowest level of facilities, and so forth. (See Farahani et al. 47] for a comprehensive review of related hierarchical facility location literature.) Within this research, the term 'hierarchical' likewise denotes different levels of facilities within an organized structure but, in our application, a demand must be serviced by at least one facility from each hierarchical level to be considered 'covered'.

The major contributions of this research are as follows. We formulate a model and develop an accompanying methodology to assess and recommend improvements to an existing regional structure designed specifically for largescale emergency response. For a multiobjective formulation, we develop a set of non-inferior solutions that allow a decision maker to assess the trade-off between maximizing coverage and minimizing modifications to the existing CRE structure. Our model also accounts for the different capabilities that are required to mitigate the impacts of a LSE through a hierarchical model. We also extend the modified CCP constraint [44] that not only prevents a facility from providing coverage to a co-located demand but also prevents coverage of all demands within a minimum stand-off distance from a facility, thereby reducing the risk to emergency response assets.

The remainder of this paper is structured as follows. In Section 2, we present 
our modeling assumptions, mathematical programming formulation, and solution method to determine optimal CRE unit locations. In Section 3, we demonstrate the implementation of our model for a scenario that is representative of a high risk CBRN incident, and we utilize the $\varepsilon$-constraint method to solve the problem. We conclude in Section 4 by summarizing the insights garnered from our analysis and identifying directions for future research.

\section{Model Formulation and Solution Methodology}

In this section, we present our model which we utilize to analyze and recommend modifications to the existing hierarchical CRE. We review the scope, approach, modeling assumptions, and math programming formulation, and we present our methodology for identifying a set of non-inferior solutions for a decision maker to consider.

\subsection{Model Formulation}

Our model focuses specifically on the initial WMD-CST, CERFP, and HRF echelons of response. Initial response constitutes the closest team at each echelon that would respond to a CBRN incident. Follow-on response is not modeled because of the additional time available to respond and the possible use of air assets to transports such follow-on units, both of which marginalize the importance of unit locations for incidents requiring a large-scale or longer-duration response.

We develop a multiobjective hierarchical extension of the MCLP which seeks to maximize coverage of the population within a defined coverage radius of response assets within each echelon while minimizing the cost of modifications, wherein we utilize the number of modifications to unit locations as a substitute for cost of modifications within this study. The hierarchical component of the problem requires a demand to be covered by each echelon of the CRE: a WMDCST, CERFP, and HRF.

Several assumptions are appropriate for a model to analyze the CRE unit locations. First, we limit the possible facility locations and demands to a finite 
set of nodes on a network. These nodes represent population centers for each county in the continental United States. Limiting the solution space reduces the complexity of the problem and frames the model around the existing U.S. road network to identify response times. Open source data from government agencies provides a realistic indication of the distances between all nodes. Furthermore, we assume a deterministic time requirement to traverse between any two nodes in the network as a function of the shortest road distance and an assumed rate of travel.

Next, we assume that the probability of a CBRN incident occurring at a given location is proportional to the location's respective population. Thus, a greater demand is generated at a more populated node. The population at a CRE unit's location also provides an indication of the risk of that CRE unit's capabilities being neutralized. We further assume that the probability of multiple simultaneous or near-simultaneous CBRN events is low and will present infrequent demand, although we do revisit this assumption via a sensitivity analysis presented in Section 3.4. Thus, we model the facilities as uncapacitated. Given the importance of rapid response, coverage is considered binary and a demand must be within a specified distance of a unit to be considered covered.

Given the scope of destruction that can occur in a CBRN incident, a CRE unit located within a minimum stand-off distance of an incident may not be able to effectively respond. Thus, we assume coverage of a location can only come from a facility located outside a minimum stand-off distance. There is no current DoD guidance or doctrine that addresses this issue, but through our analysis we intend to demonstrate the necessity to account for this risk in future decisions regarding the CRE structure.

The remaining assumptions relate to the CRE structure. First, the effectiveness of each respective WMD-CST, CERFP, and HRF is assumed to be identical and sufficient to accomplish its mission. Next, we restrict the number of WMD-CSTs, HRFs, and CERFPs to the current number in the existing enterprise. Furthermore, it is assumed each state will continue to host at least one WMD-CST. This is assumed due to the presumed political cost of removing 
an existing capability from a state. For example, in 2013 the DoD proposed disestablishing the 24th WMD-CST located in New York and 48th WMD-CST located in Florida. A number of Senators and Congressmen from both states including House Appropriations Defense Subcommittee Chairman Congressman Young (FL), Subcommittee Member Congressman Owens (NY), and Congressman Grimm (NY) fought the proposal and were able to convince Secretary of Defense Hagel to reverse the decision [48, 49].

The formulation of this problem also assumes that a WMD-CST can cover a location outside the state in which it is located. Many states have inter-state agreements already established to share CBRN resources. Additionally, WMDCSTs can be used to support other states via coordination through the National Guard Bureau, or they can be federalized to respond to an attack anywhere in the United States if approved by the appropriate authority.

We also assume that a state will not host both a HRF and a CERFP. Most states are facing challenges meeting the minimum manning requirements for the existing CERFPs. Fielding a HRF in addition to a CERFP would only exacerbate a state's personnel challenges [50]. Additionally, funding training events or activations for real world incidents for both of these units would significantly strain a state's fiscal resources.

To formulate our model, we define the following sets, parameters, and decision variables:

Sets

- $G=(N, A)$ : The underlying network.

- $N$ : Set of nodes in the network that represent possible facility locations and demands.

- $A$ : Set of undirected $\operatorname{arcs}(i, j)$ in the network, $i, j \in N, i \neq j$.

- $D$ : Set of demand nodes in the network, $D \subseteq N$.

- $S$ : Set of states $s=1, \ldots, 49$, representing the 48 Continental United States (i.e., all except for Alaska and Hawaii) and Washington D.C. 
- $N_{s} \subset N$ : Set of nodes located in state $s, s \in S$.

- $R$ : Set of FEMA regions $r=1, \ldots, 10$.

- $F_{r} \subset N$ : Set of nodes located in FEMA region $r, r \in R$.

- $L=\{w, c, h\}$ : The set of CRE echelons, where the values correspond to the WMD-CST, CERFP, and HRF echelons, respectively.

\section{Parameters}

- $h_{i}$ : Demand associated with node $i, \forall i \in D$.

- $d_{i j}$ : Distance from demand node $i$ to facility $j, \forall i \in D, j \in N$.

- $r_{\text {min }}^{\ell}$ : Minimum stand-off distance at echelon $\ell$.

- $r_{\text {max }}^{\ell}:$ Maximum coverage radius at echelon $\ell$.

- $a_{i j}^{\ell}$ : A binary parameter equal to 1 if $r_{\text {min }}^{\ell} \leq d_{i j} \leq r_{\text {max }}^{\ell}, 0$ otherwise, $\forall i \in D, j \in N$.

- $\psi_{j}^{\ell}$ : A binary parameter equal to 1 if a facility at echelon level $\ell$ currently exists at site $j, 0$ otherwise $\forall j \in N$.

- $p^{\ell}$ : The number of facilities at echelon $\ell$, that must be sited, $\forall \ell \in L$.

- $c^{\ell}$ : The cost associated with relocating a team at echelon $\ell$.

Herein, the uniformity of the relocation cost, $c^{\ell}$, for a team relocation at a given echelon is a simplifying assumption but a necessary one for this initial analysis. In practice, relocation costs will also vary geographically, as the cost to open a new facility for a team will differ with the local real estate values, labor costs, regulatory licensing fees, and/or construction material markets, and the cost to close a team's facility will include not just direct movement costs, but also costs that are more elusive to quantification such as financial costs incurred to address concerns by elected officials and citizens (e.g., holding town hall meetings, testifying before state and federal legislatures). 
Decision Variables. This model contains three linked decisions: (i) where to site facilities, (ii) how many are relocated at each echelon, and (iii) which demands to cover.

- $y_{j}^{\ell}$ : A binary decision variable equal to 1 if a facility at echelon level $\ell$ is located at site $j, 0$ otherwise, $\forall \ell \in L, j \in N$.

- $z_{i}$ : A binary decision variable equal to 1 if demand at node $i$ is covered by a facility at each echelon level, 0 otherwise, $\forall i \in D$.

- $x^{\ell}:$ An integer-valued decision variable equal to the number of teams relocated at echelon $\ell \in L$.

Given this framework, we propose the following formulation for a Maximal Conditional Covering Location Problem with Side Constraints, denoted as Problem P1:

P1: $\max \boldsymbol{f}(\boldsymbol{x}, \boldsymbol{z})=\left(f_{1}(\boldsymbol{z}), f_{2}(\boldsymbol{x})\right)$

subject to $f_{1}(\boldsymbol{z})=\sum_{i \in D} h_{i} z_{i}$

$f_{2}(\boldsymbol{x})=-\sum_{\ell \in L} c^{\ell} x^{\ell}$

$\sum_{j \in N} a_{i j}^{\ell} y_{j}^{\ell} \geq z_{i}$

$\forall \ell \in\{w, h\}, \quad i \in D$

$\sum_{j \in N}\left(a_{i j}^{c} y_{j}^{c}+a_{i j}^{h} y_{j}^{h}\right) \geq z_{i}$,

$\forall i \in D$

$\sum_{j \in N_{s}} y_{j}^{w} \geq 1$

$\forall s \in S$,

$\sum_{j \in N_{s}} y_{j}^{c}+\sum_{j \in N_{s}} y_{j}^{h} \leq 1$,

$\forall s \in S$,

$\sum_{j \in F_{r}} y_{j}^{h} \leq 1$,

$\forall r \in R$,

$\sum_{j \in N} \psi_{j}^{\ell} y_{j}^{\ell}+x^{\ell}=p^{\ell}$,

$\forall \ell \in L$,

$y_{j}^{\ell}, \in\{0,1\}$,

$\forall \ell \in L, j \in N$, 


$$
\begin{array}{ll}
z_{i} \in\{0,1\}, & \forall i \in D, \\
x^{\ell} \in \mathbb{Z}^{+}, & \forall \ell \in L .
\end{array}
$$

The objective function (1) seeks to optimize two objectives: the total demand covered (2) and the cost of unit relocations (3). Constraints (4) and (5) provide upper bounds on the decision variable $z_{i}$, wherein $z_{i}$ may only assume a value of 1 if at least one facility that can provide service at each echelon for demand $i$. Whereas Constraint (4) restricts coverage due to the respective locations of WMD-CSTs and HRFs, we present Constraint (5) separately for the CERFP echelon because CERFP-level support can be provided by either a CERFP or a HRF. (HRFs can provide both HRF and CERFP echelon coverage.) Enforcement of the minimum stand-off distance is accomplished by setting the parameter $a_{j j}=0, \forall j \in N$, and setting any $a_{i j}=0$ if the corresponding $d_{i j}$ is less than or equal to the minimum stand off distance [51. Constraint 6] requires each state to have at least one WMD-CST, and Constraint (7) prevents a state from having more than one CERFP and/or HRF. Constraint (8) limits each FEMA region to have no more than one HRF. Given a fixed number of HRFs equal to the number of FEMA regions, this is equivalent to requiring one HRF to be assigned to each FEMA region. For a given number of facilities at each echelon, $p^{\ell}$, Constraint (9) calculates the number of units being relocated, $x^{\ell}$, as a function of the number of existing unit locations that are not changed. Constraint (10) and (11) represent binary logical constraints for the facility location and coverage decision variables, and Constraint 12 restricts the number of relocations at each echelon to integer values.

\subsection{Methodology}

In lieu of solving $\mathrm{P} 1$ directly to identify the set of non-inferior solutions (e.g., with the weighting method), we utilize the $\varepsilon$-constraint method. We first 
reformulate Problem P1 to Problem P2 as follows.

$$
\text { P2: } \max \sum_{i \in D} h_{i} z_{i}
$$

subject to Constraints (4)- 12 ,

$$
\sum_{\ell \in L} c^{\ell} x^{\ell} \leq \varepsilon, \quad \forall \ell \in L
$$

In this reformulation, we replaced the objective function (1) with the objective of maximizing the total demand covered (13). We impose Constraint (14) to bound our second objective, the minimization of costs due to moving units, to be no more than $\varepsilon$, an allowed cost of relocations. We initially set $\varepsilon=0$ and solve P2 to identify the coverage level for the current CRE unit locations. By iteratively increasing the value of $\varepsilon$ and re-solving P2, we develop a set of noninferior solutions that highlight the trade-off between maximizing coverage and minimizing cost.

\section{Computational Results}

In this section we demonstrate the application of our model. We first review the data used in the model and our solution technique. Next, we examine the current level of coverage provided by the existing CRE unit locations and compare it to an optimal level of coverage when all units are allowed to relocate. We then examine the trade-off between cost and coverage considering an independent view of each echelon and an enterprise approach, respectively. The independent view examines coverage at each echelon as if coverage depends only on the echelon under examination. The enterprise approach assumes coverage is dependent upon all three echelons, and a demand is only considered covered if it is covered by all three echelons. Finally, we examine additional advantages of the CCP constraint.

The data used in this analysis was compiled from unclassified open-source resources; it provides an approximation of the true system but is not an exact representation. The road infrastructure network utilized consists of 3109 
nodes that represent the population centers for counties in the Continental United States and the District of Columbia. The arc length between nodes was determined using U.S. highway road distance from the Center for Transportation Analysis Oak Ridge National Highway Network (NHN) [52]. Using this network, we applied the Floyd-Warshall Algorithm [53] to determine the shortest path length between each pair of nodes, set this equal to $d_{i j}$ and, for $d_{i j} \in\left[r_{\min }^{\ell}, r_{\max }^{\ell}\right]$, set $a_{i j}^{\ell}=1$ and 0 otherwise for each echelon $\ell \in L$.

Population estimates for each county, $h_{i}$, are taken from the 2010 U.S. Census [54. Counties were classified by the CDC's 2013 NCHS Urban-Rural Classification Scheme for Counties [55] and are depicted in Figure 1. CRE unit locations [56] were estimated to exist at the respective population centers of the counties in which they are located and do not depict their precise locations.

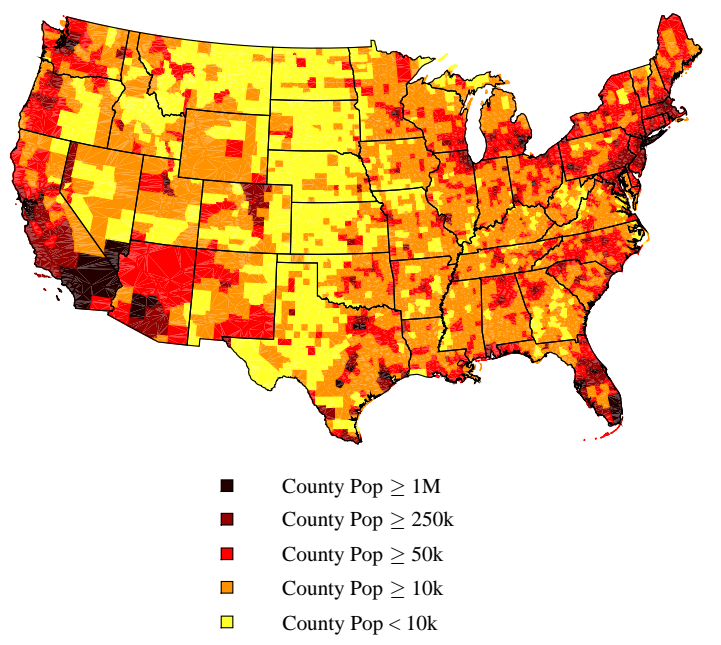

Figure 1: U.S. Population by Counties

The maximum coverage radius for units within each echelon, $r^{\ell}$, was developed using existing DoD doctrine and accepted standards within the DoD. Response time requirements were converted to distance requirements using an assumed 50 miles per hour response speed. Thus, WMD-CSTs are assumed to have a maximum response time of five hours, which is equivalent to a maximum 
coverage distance of 250 miles. CERFPs are assumed to have a six-hour response requirement, which equates to a 300-mile maximum coverage distance. Finally, HRFs are assumed to have a 12-hour response requirement which corresponds to a 600-mile maximum coverage radius. The minimum stand-off distance was developed using the projected radius of effects from a 10 kiloton nuclear device detonated at ground level, which is recognized as the most likely nuclear device to be used in a domestic terror attack [57. The significant damage from this device is estimated to occur within a three-mile radius, and the deadly fallout radius is estimated to extend for 10-20 miles. Given this information, we define a conservative minimum standoff distance of 25 miles. A summary of the minimum and maximum coverage radii at each echelon is provided in Table 1 .

Table 1: The minimum and maximum coverage radii at each echelon

\begin{tabular}{ccc} 
Echelon $(\ell)$ & $r_{\min }^{\ell}$ & $r_{\max }^{\ell}$ \\
\hline$w$ & 25 & 250 \\
$c$ & 25 & 300 \\
$h$ & 25 & 600 \\
\hline
\end{tabular}

Solutions were found for P2 instances using a computer having an Intel Xeon Processor E5-1620 and 32 GB of memory by invoking the commercial solver CPLEX (Version 12.6) [58] from Matlab R2014a. All solutions reported are within a relative optimality tolerance of $0.49 \%$. This tolerance was selected to prevent excessive run times; both the MCLP and the CCP are known NPHard problems [59, and certain instances of our problem were not solvable to optimality within up to 24 hours of computational effort.

\subsection{Current and Maximal Coverage}

The current CRE unit locations cover $83 \%$ of the population. Although the information used in our analysis is not classified, we judiciously refrain from providing herein the locations of the areas and populations not supported within the specified response times by the current CRE unit locations and dispositions. 
It is also worth noting the current unit locations are estimates and do not represent exact locations; thus, our model provides an approximation of the true coverage. Our goal was to demonstrate the usefulness of the methodology to warrant developing a refined data set with true unit locations and feasible facility locations.

If we allow all units to relocate by solving P2 in the absence of Constraint (14), we find that optimal CRE unit locations can cover $99.47 \%$ of the population, as depicted in Figure 2, an improvement of over 49 million people covered compared to current unit locations.

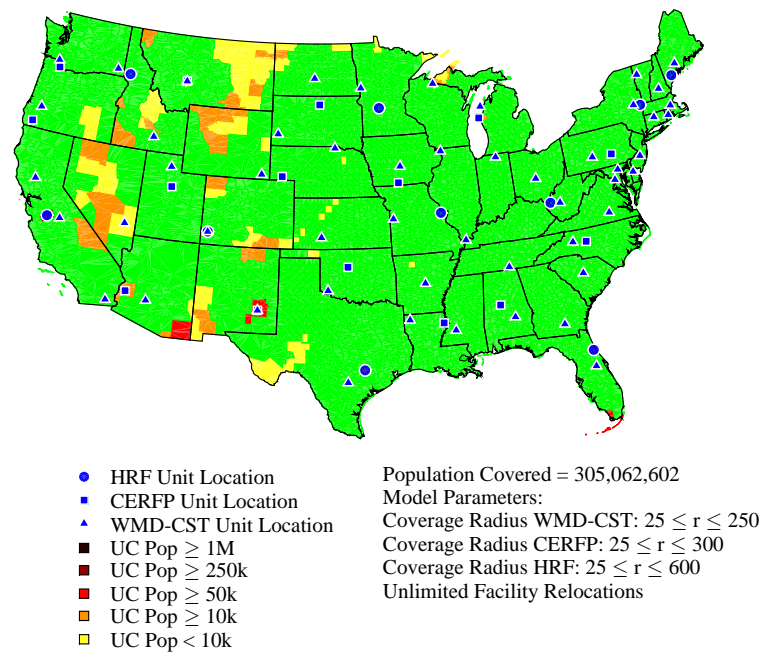

Figure 2: CRE Unit Locations for Maximal Coverage

We note that maximizing coverage without a tight bound on costs may yield a solution that is not necessarily non-inferior with respect to the minimization of costs, as alternative maximal covering solutions may exist that have lower costs. To identify the extreme point on the Pareto frontier, one may either adopt a preemptive programming approach by subsequently affixing the coverage level as a constraint and minimizing the relocation costs, or perform a line search on $\varepsilon$ to find the smallest value for which an decrease does not yield a solution that reduces the population coverage of the CRE. We applied the latter methodology 
and subsequently identified the solution depicted in Figure2, which requires the relocation of $53 \mathrm{CRE}$ units, as having no alternative optima with lower costs (i.e., it is a non-inferior solution).

\subsection{Independent View of Each Echelon}

We begin our detailed analysis of the trade-off between coverage and cost (herein, represented by the total number) of modifications to the structure by first adopting an independent view of each echelon. As we examine each respective echelon, we assume the other echelons provide full coverage, and thus coverage is only dependent on the echelon under consideration. This independent view identifies the effectiveness of each echelon and the minimum number of modifications required at each echelon to attain a specified coverage threshold. By comparing independent views we can establish an upper bound on coverage for a given number of relocations at each echelon. In this analysis we seek to obtain $98 \%$ coverage as a goal for each echelon, but other minimum coverage standards could be considered.

Figure 3 depicts the attainable coverage as the allowable number of HRF relocations increases from 0 to 10 and indicates that, if we desire to attain a coverage level of $98 \%$ for the entire enterprise, we need to relocate at least three HRFs.

The CERFP echelon analysis is more nuanced than the HRF echelon because HRFs can provide both HRF and CERFP echelon coverage. Thus, we vary the allowable CERFP relocations from 0 to 15, and the allowable HRF relocations from 0 to 10, for a total of 150 alternatives. Figure 4 depicts the influence of the CERFP echelon on coverage. We note that HRF relocations have a significant impact upon CERFP coverage when relocating up to four HRFs. For example, if we relocate three HRFs (i.e., the number of relocations required to attain $98 \%$ coverage for the HRF echelon), at least five CERFPs must be relocated to attain greater than $98 \%$ coverage for the CERFP echelon. If we instead relocate no HRFs, then we must relocate 10 CERFPs to cover $98 \%$ of the population. Table 2 reports, for each possible number of allowed HRF relocations, the minimum 


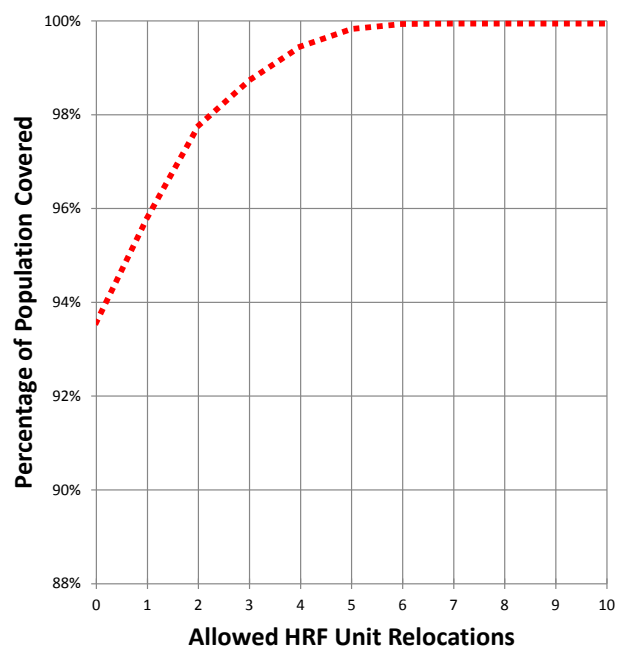

HRF Echelon Coverage

Figure 3: Independent View of Coverage at the HRF Echelon

number of CERFPs that must also be relocated to attain at least a $98 \%$ coverage level at the CERFP echelon.

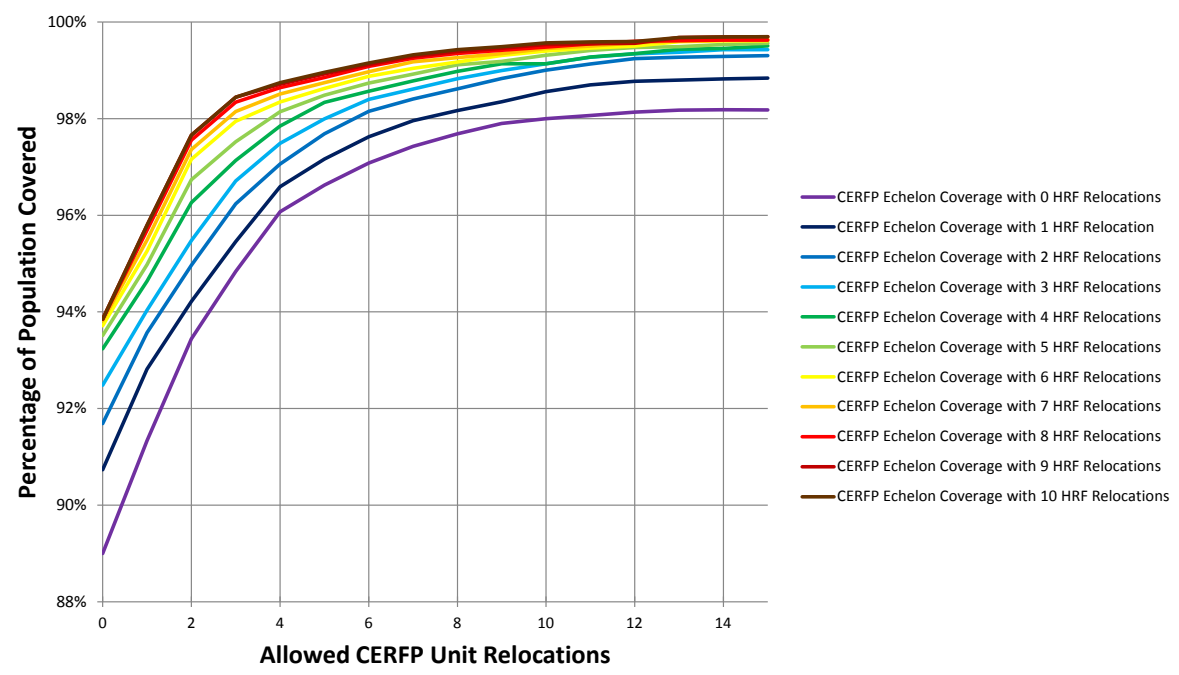

Figure 4: Independent View of Coverage at the CERFP Echelon

Finally, we analyze the WMD-CST echelon independently as depicted in 
Table 2: Required CERFP relocations to attain $98 \%$ coverage for a given number of HRF relocations

\begin{tabular}{cc} 
HRF relocations & Required CERFP relocations \\
\hline 0 & 10 \\
1 & 8 \\
2 & 6 \\
$3-4$ & 5 \\
$5-6$ & 4 \\
$7-10$ & 3 \\
\hline
\end{tabular}

Figure 5 At least seven WMD-CST relocations are required to attain $98 \%$ coverage at the WMD-CST echelon.

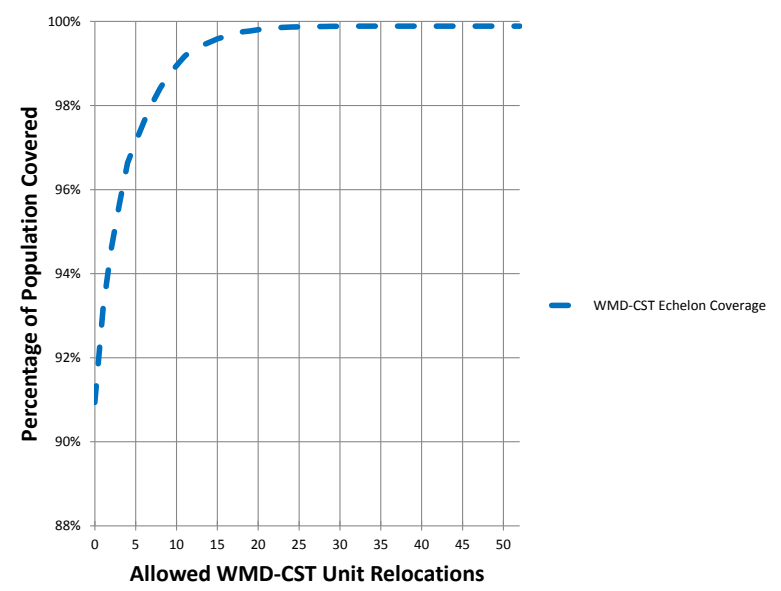

Figure 5: Independent View of Coverage at the WMD-CST Echelon

Overlaying each independent view, Figure 6 illustrates the upper bound on attainable coverage for a specified number of relocations at each echelon. Figure 6 also allows a decision maker to visualize how coverage is impacted independently at each echelon. For example, to attain greater than $98 \%$ coverage, we need to relocate at least three HRFs, five CERFPs, and seven WMD-CSTs. Although an examination of each echelon independently indicates such a solu- 
tion can attain coverage for up to $98 \%$ of the population, if we maintain the same objective function and constraints while allowing at most three HRF, five CERFP, and seven WMD-CST relocations, respectively, we actually only cover 95.44\% of the population when considering the enterprise view. This difference exists because specific counties covered by each echelon differ. Thus, we observe that modifying the echelons independently does not accomplish a $98 \%$ coverage threshold for the entire enterprise and, to do so, we must treat coverage from a holistic perspective.

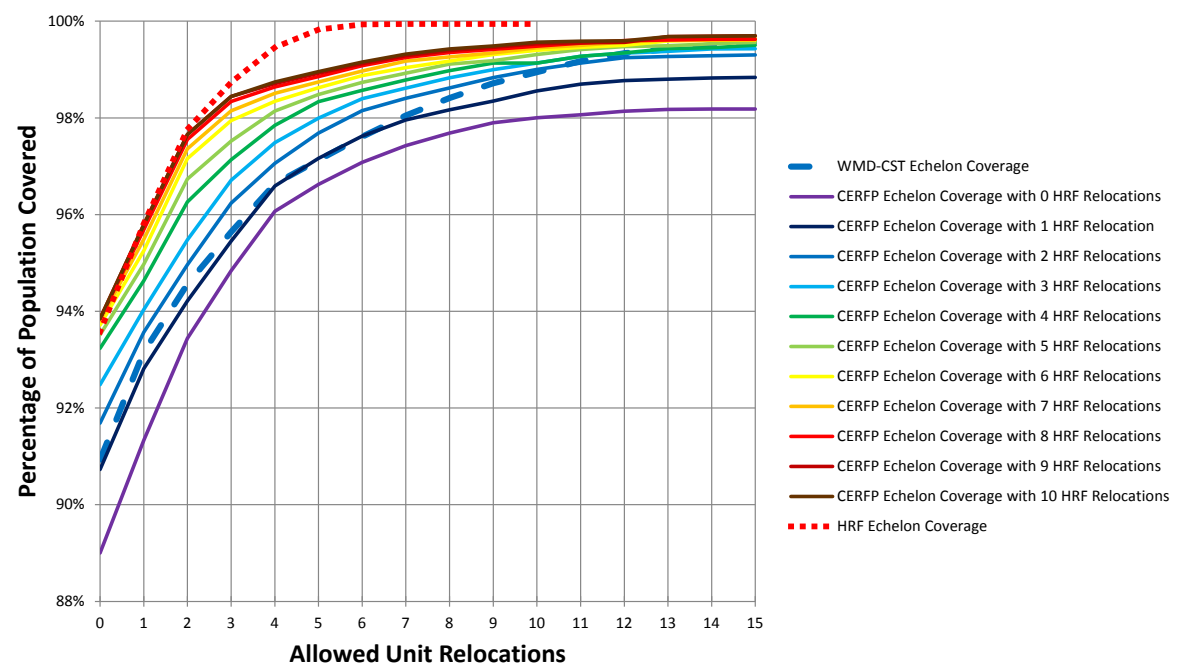

Figure 6: Independent View of Coverage for each Echelon

\subsection{Enterprise Approach}

We now assess the trade-off between coverage and modifications from a enterprise perspective. There are over 9300 possible combinations for how many CRE units among the various echelons may relocate but, if we assume that minimizing the total number of modifications to the CRE is also desirable, there are only 54 alternatives in the non-inferior set. This non-inferior set is developed by iteratively incrementing the total number of allowed relocations (from 0 to 53) and maximizing coverage for each iteration, as depicted in Figure 7. This 
reduced subset of alternatives contains the only solutions of practical interest to a decision maker and significantly reduces the computation time required.

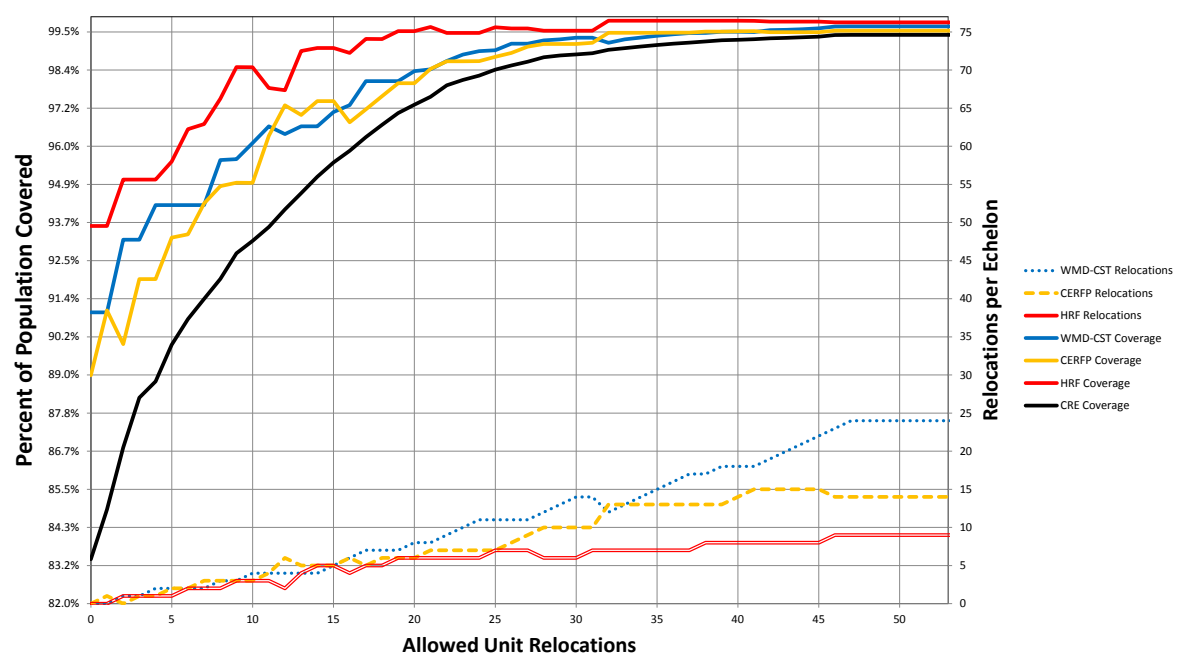

Figure 7: Percentage of population covered as the number of allowed relocations increases

Figure 7 identifies the coverage of the entire CRE, the coverage at each echelon, and the corresponding number of unit relocations that occur within each echelon for a total number of allowed relocations. The non-inferior set of solutions is represented by the coverage of the entire CRE (i.e., "CRE Coverage"), for which the coverage level is indicated on the left axis of the figure. The CRE coverage is non-decreasing in $\varepsilon$. Such a monotonic relationship is not present for any of the three independent echelons, as represented respectively by the top three plots in Figure 7. For example, as $\varepsilon$ increases from 10 to 11, the CERFP and WMD-CST coverages increase, but the HRF coverage decreases, yielding an overall increase in the CRE coverage. Thus, accommodations are made between echelons for a given relocation budget to maximize the overall CRE coverage. The lower three plots in Figure 7 indicate the number of unit relocations required within each echelon, as indexed on the right axis of the figure, to attain the reported CRE coverage. Whereas the WMD-CST echelon predominantly requires the largest number of relocations over the range of $\varepsilon$-values examined, 
when considering the per capita moves at each echelon, the CERFP and HRF echelons require a much greater modification to their respective echelon structure to improve CRE coverage than the WMD-CST echelon. For example, when 20 relocations are allowed, a total of 8 WMD-CSTs, 6 CERFPs, and 6 HRFs are relocated, which respectively represent a $15 \%, 40 \%$, and $60 \%$ modification to each echelon.

\subsection{Sensitivity Analysis of Selected Parameters}

Herein, we examine the resiliency of the proposed solution for CRE unit locations in Figure 2 by revisiting two key assumptions. First, our baseline analysis assumed a travel rate of 50 miles per hour. Second, our examination considered a CBRN event as an LSE; that is, an attack will occur at a single location. We postulate the first assumption to be reasonable because, although a CBRN event will induce traffic congestion, the CRE units will travel in a direction counter to the congested flow of vehicles evacuating and/or fleeing the site of the attack. Regarding the second assumption, the development of CBRN attack capability requires specialized knowledge and materials, making it more challenging to mount an attack without detection. To minimize the likelihood of detection, a hostile agent will reasonably concentrate efforts towards mounting a successful CBRN attack against one target.

However, we find it worthwhile to revisit both of these assumptions to assess the resiliency of the solution proposed in Figure 2 For the recommended CRE unit locations, we analyze the performance of the system for a travel rate reduced from 50 down to 20 miles per hour, in 5 mile-per-hour increments. Reported in Table 3 are the corresponding coverages (\%) for these degraded travel rates for the recommended solution shown in Figure2 2 as optimized for a 50 mile per hour travel rate.

The sensitivity analysis depicted in Table 3 illustrates that both the optimal and the current solutions' coverages degrade rapidly with a decrease in the assumed unit response speed. This intuitive result confirms the importance of the assumed response speed. During a response to a CBRN event, it may 
Table 3: Comparison of Coverage (\%) for the Optimal Solution Depicted in Figure 2 and the Solution using Current CRE Unit Locations for Degraded Response Speeds

Optimal Solution Current Solution

\begin{tabular}{ccc} 
Response Speed $(\mathbf{m p h})$ & Coverage $(\boldsymbol{\%})$ & Coverage $(\%)$ \\
\hline 50 & 99.5 & 83.4 \\
45 & 85.3 & 74.8 \\
40 & 67.7 & 65.7 \\
35 & 49.5 & 55.0 \\
30 & 33.6 & 43.0 \\
25 & 17.8 & 31.3 \\
20 & 8.1 & 18.6 \\
\hline
\end{tabular}

be necessary to dedicate resources (e.g., state highway police, local police) to ensure that travel routes are not congested to facilitate uninhibited response by critical assets such as CRE units. The results in Table 3 further demonstrate that the percentage of the population covered by the optimal solution depicted in Figure 2 is less robust to reductions in the assumed response speed, relative to the solution using current CRE unit locations. This result is also expected; the maximization of coverage is attained by relocating CRE units in a manner that more efficiently utilizes their full capability, which includes covering populations up to 250-miles away. This comparison further reinforces the need for planned, proactive, and rehearsed traffic control measures to facilitate rapid response efforts in the aftermath of a CBRN event.

We further consider a hostile agent mounting two, three, four, or five simultaneous attacks instead of one attack, and we examine the likelihood of the optimal and current CRE unit locations being sufficient to provide dedicated response at each echelon within the CRE. For each number of simultaneous attacks, we conducted 1,000 simulations of attack locations, wherein the probability of a location being attacked is proportional to the size of its population and for which we do not allow attacks to be co-located within a simulation. Reported in Table 4 are the likelihoods of a CBRN attack having a dedicated CRE unit for response, at each echelon level. 
Table 4: Simulated Likelihood (\%) of a CBRN Incident having a Dedicated CRE Response Unit, by Echelon, for both the Optimal Solution depicted in Figure 2 and the Solution using Current CRE Unit Locations

Number of

\begin{tabular}{ccccccc}
$\begin{array}{c}\text { Simultaneous } \\
\text { Attacks }\end{array}$ & \multicolumn{2}{c}{ WMD-CST } & \multicolumn{2}{c}{ CERFP } & \multicolumn{2}{c}{ HRF } \\
\hline 1 & Optimal & Current & Optimal & Current & Optimal & Current \\
2 & 99.9 & 91.6 & 99.7 & 88.9 & 99.8 & 94.3 \\
3 & 99.5 & 90.9 & 97.7 & 88.4 & 98.2 & 92.4 \\
4 & 99.1 & 90.9 & 96.9 & 88.4 & 97.0 & 91.9 \\
5 & 99.0 & 89.6 & 95.3 & 86.6 & 95.5 & 89.7 \\
\hline
\end{tabular}

The simulation results presented in Table 4 indicate that both the optimal and current solutions are relatively resilient. Under the range of the number of simultaneous attacks considered, the coverage of the optimal solution suffered a degradation of up to $1.0 \%, 5.3 \%$ and $6.2 \%$ for the likelihood of a CBRN attack having dedicated support at the WMD-CST, CERFP, and HRF levels, respectively. For the solution using current CRE unit locations, the maximum degradations in coverage under these simulated conditions are $2.0 \%, 2.3 \%$ and $5.7 \%$ at the respective WMD-CST, CERFP, and HRF echelons. Of note, the optimal solution under the simulated conditions improves upon the likelihood of the current solution to provide dedicate support by an average of $8.5 \%, 8.8 \%$, and $5.4 \%$ (and a minimum of $8.1 \%, 6.5 \%$, and $5.0 \%$ ) at the WMD-CST, CERFP, and HRF levels, respectively. Thus, we find the optimal solution to be an improvement over the current CRE unit locations with regard to its performance under small numbers of multiple simultaneuous CBRN attacks.

\subsection{Second-order Effects of the Conditional Coverage Restriction}

Finally, we consider two additional measures that are not objectives of our formulation but which further demonstrate the value of incorporating the conditional covering constraint. First, we examine the amount of population colocated with each CRE unit as a measure of risk. If we assume the probability of a CBRN event taking place in a county to be proportional to the population 
of that county, then a co-located CRE unit incurs a similar relative likelihood of an attack at its location. Thus, the risk of a CRE unit's capabilities being neutralized by a CRBN event is proportional to the size of the co-located population. Over the range of $\varepsilon \in\{0, \ldots, 53\}$, Figure 8 depicts the decrease in population that is co-located with CRE units when the CCP constraint is enforced, relative to the solution attained in the absence of such a restriction. In the best performing case (i.e., $\varepsilon=15$ ), this yields a benefit of almost 62 million fewer people co-located with CRE units. Even a $5 \%$ reduction in co-located population corresponds to over 16 million fewer people, thereby reducing the risk to the CRE for most values of $\varepsilon$.

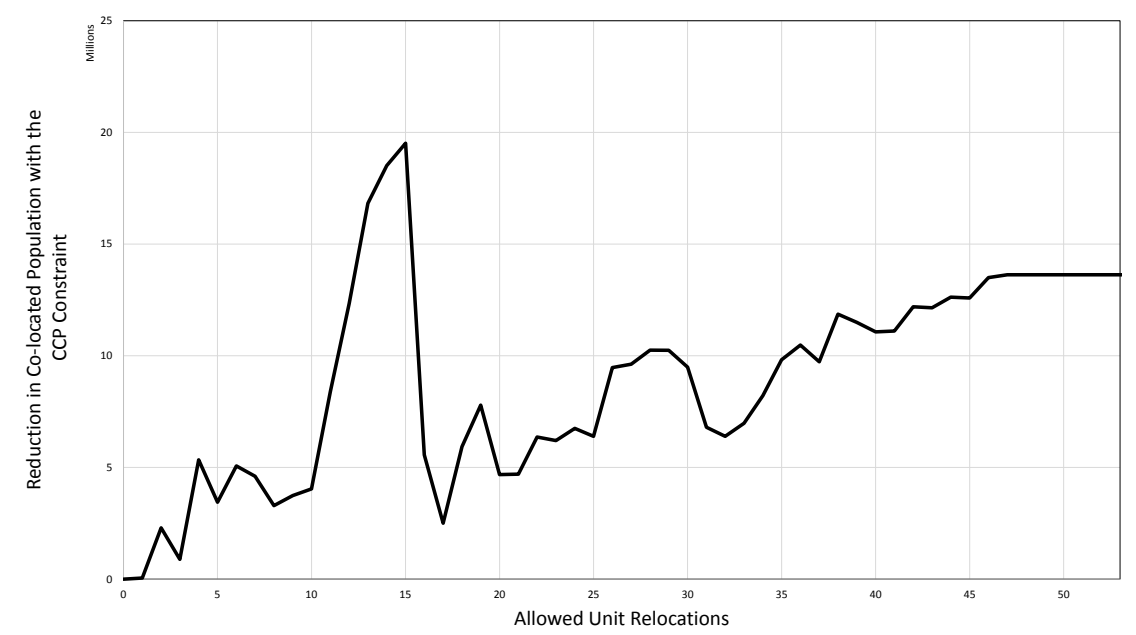

Figure 8: Reduction in population co-located with CRE units using the CCP constraint

The higher levels of co-located populations depicted in Figure 8 over the range of $10 \leq \varepsilon \leq 15$ are not the direct result of our formulation or its optimal solution(s); it instead results from a comparison to the optimal solution attained in the absence of the conditional covering constraint. Without such a constraint, the optimal coverage attained when increasing from 10 to 11 allowable unit relocations relocates CRE units to Phoenix, Orange County (California), and Salt Lake City. When the number of allowable unit relocations is further increased, the optimal solution in the absence of the conditional cover- 
ing constraint relocates teams to Los Angeles for 12 relocations; Indianapolis, Tampa, and Columbus (Ohio) for 13 relocations; and Brooklyn for 14 relocations. Of note, the relative reduction in the co-located population decreases beyond 15 allowable unit relocations because the solution in the absence of the CCP constraint no longer relocates units to Los Angeles or Phoenix for 16 relocations, and no longer relocates units to Brooklyn or Columbus for 17 relocations. In general, the increased co-location of units in larger cities results from the network topology; large cities are often the crossroads for major highways, especially outside of the Northeast megalopolis, and therefore offer well suited locations for maximizing the coverage radius for a team. When the CCP constraint is not included, there is no drawback to relocating units to these densely populated areas. With the CCP constraint, however, the increased number of populations within the covering radius of teams located at the intersections of major highways is not offset by the loss coverage capability for the co-located populations.

The second measure assesses the redundancy of coverage by evaluating the percentage of the population that is covered by at least two teams or "double covered". Figure 9 illustrates the increase in the population that is double covered with the imposition of the CCP constraint relative to the optimal solution attained in its absence.

The relative increase in double coverage directly relates to the invocation of the CCP constraint. Without the constraint, there is no incentive for a solution to double-cover any demands; the problem formulation favors a reduction in overlapping coverage to maximize the covered population. With the CCP constraint, overlapping coverage by CRE units is required. Given a CRE unit within a specified echelon, the population with which it is co-located can only be covered by a different CRE unit within the same echelon that is located sufficiently distant but also within the covering radius. This yields a mutual reinforcement between $\mathrm{CRE}$ units and results in both of the populations that have a co-located CRE unit being covered by the non-adjacent CRE unit, and further results in any populations within the radius of both CRE units being 


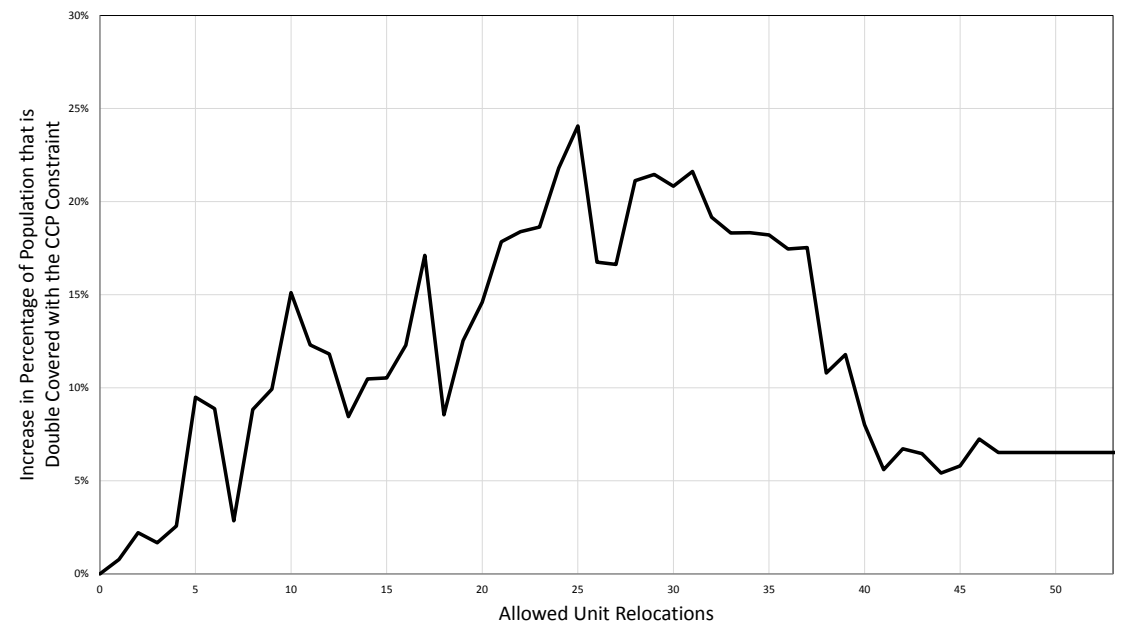

Figure 9: Increase in percentage of population that is double covered when using the CCP constraint

double covered. For high population density regions that are the most likely targets of an attack, the effect of double coverage resulting from mutually reinforcing CRE units impacts larger populations, which consequently improves the resiliency of the system, thereby increasing the amount of the total population that can be supported by multiple units should an LSE require it.

\section{Conclusions and Recommendations}

In this paper we demonstrated a methodology for modifying the existing CRE structure. Our multiobjective approach maximizes the use of the existing structure, defines trade-offs between competing objectives, and significantly reduces the set of alternatives to consider. We demonstrated that in the absence of resource constraints, we can improve coverage by more than $15 \%$, approximately 49 million people. Furthermore, with only 23 unit relocations (less than a $30 \%$ modification of the entire structure) coverage will exceed 98\%, an improvement of an additional 45 million people covered. Finally, we demonstrated that the enforcement of the CCP constraint reduces the risk of an LSE neutralizing CRE assets and improves redundancy of coverage. 
Potential improvements to our model merit further study. First, our use of county-based network aggregates the Continental U.S. population to 3109 possible locations, which may overly simplify the number of possible facility locations. Counties also vary greatly in size, resulting in nodes in our network model representing varying area sizes. One possible alternative to county-level aggregation is to establish a network with population nodes corresponding to postal zip codes, which entail over 40,000 nodes. Whereas this expansion of the instance size would provide result in a network having a higher granularity representation of the population, it also would increase the complexity of the problem, which would likely preclude the use of an exact solution method and require the development of a customized heuristic. Second, our model assumed that a CRE unit could be relocated to any node (i.e., county) in the network. Depending on the availability of real estate and facilities to base such units, a modeling extension might consider a modification of possible facility locations to only consider sites predetermined by a governing authority. Finally, it merits mention that societies are not egalitarian; some population areas merit a higher level of protection due to their contribution to critical sectors of a national economy and/or governance, as these locations are more likely to incur a CBRN attack. As such, a modeling extension should also examine a third objective: minimizing either the average or the maximum response time for a subset of CBRN targets that are weighted by importance. Thus, the resulting model would seek to balance service quality to priority nodes, total coverage, and the cost of changing the existing enterprise unit locations.

\section{Disclaimer}

The views expressed in this article are those of the authors and do not reflect the official policy or position of the United States Air Force, the United States Army, the Department of Defense, or the United States Government. 


\section{Acknowledgment}

The authors gratefully thank the Area Editor, the Associate Editor, and two reviewers for their constructive comments that have helped improve the presentation of this paper.

\section{References}

[1] Office of the Secretary of Defense, Strategy for homeland defense and defense support of civil authorities, Tech. rep. (2013).

URL http://www .defense.gov/news/homelanddefensestrategy ·pdf

[2] The Joint Staff, Joint Publication 3-41: Chemical, Biological, Radiological, and Nuclear Consequence Management, Department of Defense (June 2012).

URL http://www.dtic.mil/doctrine/new_pubs/jp3_41.pdf

[3] J. A. St. Laurent, Homeland defense and weapons of mass destruction: Additional steps could enhance the effectiveness of the National Guard's life-saving response forces, Tech. Rep. GAO-12-114, The U.S. Government Accountability Office (2011).

URL http://www.gao.gov/new.items/d12114.pdf

[4] H. Jia, F. Ordóñez, M. Dessouky, A modeling framework for facility location of medical services for large-scale emergencies, IIE Transactions 39 (1) (2007) 41-55.

[5] P. Kolesar, W. E. Walker, An algorithm for the dynamic relocation of fire companies, Operations Research 22 (2) (1974) 249-274.

[6] K. Sudtachat, M. E. Mayorga, L. A. Mclay, A nested-compliance table policy for emergency medical service systems under relocation, Omega 58 (2016) 154-168. 
[7] N. R. Paul, Optimizing the domestic chemical, biological, radiological, and nuclear response enterprise, Master's thesis, Air Force Institute of Technology (2015).

[8] R. Church, C. ReVelle, The maximal covering location problem, Papers in Regional Science 32 (1) (1974) 101-118.

[9] M. S. Daskin, A maximum expected covering location model: Formulation, properties and heuristic solution, Transportation Science 17 (1) (1983) 4870.

[10] C. Toregas, R. Swain, C. ReVelle, L. Bergman, The location of emergency service facilities, Operations Research 19 (6) (1971) 1363-1373.

[11] A. B. Calvo, D. H. Marks, Location of health care facilities: An analytical approach, Socio-Economic Planning Sciences 7 (5) (1973) 407-422.

[12] R. Z. Farahani, N. Asgari, N. Heidari, M. Hosseininia, M. Goh, Covering problems in facility location: A review, Computers \& Industrial Engineering 62 (1) (2012) 368-407.

[13] S. Belardo, J. Harrald, W. A. Wallace, J. Ward, A partial covering approach to siting response resources for major maritime oil spills, Management Science 30 (10) (1984) 1184-1196.

[14] V. Dokmeci, A multiobjective model for regional planning of health facilities, Environment and Planning A 11 (5) (1979) 517-525.

[15] K. Hogan, C. ReVelle, Concepts and applications of backup coverage, Management Science 32 (11) (1986) 1434-1444.

[16] J. Current, H. Min, D. Schilling, Multiobjective analysis of facility location decisions, European Journal of Operational Research 49 (3) (1990) 295-307.

[17] U. Bhattacharya, J. Rao, R. Tiwari, Bi-criteria multi facility location problem in fuzzy environment, Fuzzy Sets and Systems 56 (2) (1993) 145-153. 
[18] J. W. George, C. S. ReVelle, Bi-objective median subtree location problems, Annals of Operations Research 122 (1-4) (2003) 219-232.

[19] J. G. Villegas, F. Palacios, A. L. Medaglia, Solution methods for the biobjective (cost-coverage) unconstrained facility location problem with an illustrative example, Annals of Operations Research 147 (1) (2006) 109141.

[20] M. da Graça Costa, M. E. Captivo, J. Clímaco, Capacitated single allocation hub location problem: A bi-criteria approach, Computers \& Operations Research 35 (11) (2008) 3671-3695.

[21] E. Carrizosa, A. Ushakov, I. Vasilyev, Threshold robustness in discrete facility location problems: a bi-objective approach, Optimization Letters (2015) 1-18.

[22] J. R. Current, C. ReVelle, J. L. Cohon, The maximum covering/shortest path problem: A multiobjective network design and routing formulation, European Journal of Operational Research 21 (2) (1985) 189-199.

[23] S. Bhaskaran, M. A. Turnquist, Multiobjective transportation considerations in multiple facility location, Transportation Research Part A: General 24 (2) (1990) 139-148.

[24] S. Chanta, M. E. Mayorga, L. A. McLay, Improving emergency service in rural areas: a bi-objective covering location model for EMS systems, Annals of Operations Research 221 (1) (2014) 133-159.

[25] G. Leo, A. Lodi, P. Tubertini, M. Di Martino, Emergency department management in Lazio, Italy, Omega 58 (2016) 128-138.

[26] S. Khodaparasti, H. Maleki, M. Bruni, S. Jahedi, P. Beraldi, D. Conforti, Balancing efficiency and equity in location-allocation models with an application to strategic EMS design, Optimization Letters (2015) 1-18. 
[27] R. Z. Farahani, M. SteadieSeifi, N. Asgari, Multiple criteria facility location problems: A survey, Applied Mathematical Modelling 34 (7) (2010) 16891709.

[28] Z.-H. Zhang, H. Jiang, A robust counterpart approach to the bi-objective emergency medical service design problem, Applied Mathematical Modelling 38 (3) (2014) 1033-1040.

[29] S. Rath, W. J. Gutjahr, A math-heuristic for the warehouse locationrouting problem in disaster relief, Computers \& Operations Research 42 (2014) 25-39.

[30] M. Ehrgott, X. Gandibleux, A survey and annotated bibliography of multiobjective combinatorial optimization, OR-Spektrum 22 (4) (2000) 425-460.

[31] J. L. Cohon, Multiobjective programming and planning, Dover Publications Inc., Mineola, NY, 2013.

[32] M. Ehrgott, Multicriteria optimization, Springer Science \& Business Media, 2006.

[33] J.-F. Bérubé, M. Gendreau, J.-Y. Potvin, An exact $\varepsilon$-constraint method for bi-objective combinatorial optimization problems: Application to the traveling salesman problem with profits, European Journal of Operational Research 194 (1) (2009) 39-50.

[34] J. E. Bell, S. E. Griffis, W. A. Cunningham, J. A. Eberlan, Location optimization of strategic alert sites for homeland defense, Omega 39 (2) (2011) $151-158$.

[35] G. Mavrotas, Effective implementation of the $\varepsilon$-constraint method in multiobjective mathematical programming problems, Applied Mathematics and Computation 213 (2) (2009) 455-465.

[36] V. Chankong, Y. Y. Haimes, Multiobjective decision making: Theory and methodology, Noth-Holland, Amsterdam, 1983. 
[37] J. L. Redondo, J. Fernández, J. D. Á. Hervás, A. G. Arrondo, P. M. Ortigosa, Approximating the pareto-front of a planar bi-objective competitive facility location and design problem, Computers \& Operations Research 62 (2014) 337-349.

[38] H. Jia, F. Ordóñez, M. M. Dessouky, Solution approaches for facility location of medical supplies for large-scale emergencies, Computers \& Industrial Engineering 52 (2) (2007) 257-276.

[39] P. Murali, F. Ordóñez, M. M. Dessouky, Facility location under demand uncertainty: Response to a large-scale bio-terror attack, Socio-Economic Planning Sciences 46 (1) (2012) 78-87.

[40] M. Peker, B. Y. Kara, The $P$-hub maximal covering problem and extensions for gradual decay functions, Omega 54 (2015) $158-172$.

[41] J.-B. Sheu, Dynamic relief-demand management for emergency logistics operations under large-scale disasters, Transportation Research Part E: Logistics and Transportation Review 46 (1) (2010) 1-17.

[42] R. Abounacer, M. Rekik, J. Renaud, An exact solution approach for multiobjective location-transportation problem for disaster response, Computers \& Operations Research 41 (2014) 83-93.

[43] I. D. Moon, S. S. Chaudhry, An analysis of network location problems with distance constraints, Management Science 30 (3) (1984) 290-307.

[44] B. J. Lunday, J. C. Smith, J. B. Goldberg, Algorithms for solving the conditional covering problem on paths, Naval Research Logistics (NRL) 52 (4) (2005) 293-301.

[45] R. Huang, S. Kim, M. B. Menezes, Facility location for large-scale emergencies, Annals of Operations Research 181 (1) (2010) 271-286.

[46] S. C. Narula, U. I. Ogbu, An hierarchal location-allocation problem, Omega 7 (2) (1979) $137-143$. 
[47] R. Z. Farahani, M. Hekmatfar, B. Fahimnia, N. Kazemzadeh, Hierarchical facility location problem: Models, classifications, techniques, and applications, Computers \& Industrial Engineering 68 (2014) 104-117.

[48] Florida National Guard Department of Military Affairs, Florida's WMD disaster response unit saved from elimination, last accessed on 14 January 2014.

URL http://dma.myflorida.com/floridas-wmd-disaster-responseunit-saved-from-elimination/

[49] C. Schumer, K. Gillibrand, M. Grimm, P. Tonko, B. Nelson, M. Rubio, B. Young, Schumer, Gillibrand, Grimm urge DoD \& National Guard to protect terror response teams in New York, including Fort Hamilton team in Brooklyn, last accessed on 14 January 2014.

URL http://www.gillibrand.senate.gov/newsroom/press/release/ schumer-gillibrand - grimm - urge - dod - and - national - guard - to protect-terror-response-teams - in-new-york-including-forthamilton-team-in-brooklyn

[50] D. M. D'Agostino, Homeland defense: Preliminary observations on defense chemical, biological, radiological, nuclear, and high-yield explosives consequence management plans and preparedness, Tech. Rep. GAO-09-927T, The U.S. Government Accountability Office (2009).

URL http://www.gao.gov/products/GAO-09-927T

[51] B. J. Lunday, The modified covering problem on paths and trees, Master's thesis, The Univeristy of Arizona (2001).

[52] Center for Transportation Analysis, County-to-county distance matrix, last accessed on 14 January 2014 (2010).

URL http://cta.ornl.gov/transnet/SkimTree.htm

[53] R. L. Francis, J. A. White, L. F. McGinnis, Facility layout and location: An analytical approach, Vol. 31, Prentice-Hall, Englewood Cliffs, NJ, 1974. 
[54] US Census Bureau, 2010 US population by county, last accessed on 14 January 2014 (2010).

URL http://www2 . census .gov/geo/tiger/TIGER2010DP1/County_ 2010Census_DP1.zip

[55] 2013 National Center for Health Statistics Urban-Rural Classification Scheme for Counties, Tech. rep., US Department of Health and Human Services (2014).

[56] Center for Army Lessons Learned, Catastrophic disaster response staff officer's handbook, last accessed on 14 January 2014 (2006).

URL http://usacac. army.mil/cac2/call/docs/06-08/ap-h.asp

[57] National Security Staff Interagency Policy Coordination Subcommittee, Planning guidance for response to a nuclear detonation, Tech. rep. (2010). URL http://www.epa.gov/radiation/docs/er/planning-guidancefor-response-to-nuclear-detonation-2-edition-final.pdf

[58] International Business Machines, IBM ILOG CPLEX Optimization Studio V12.6.0 documentation, last accessed 15 April 2015 (2015).

URL http://wwW-01.ibm . com/support/knowledgecenter/SSSA5P_ 12.6.0/ilog.odms.studio.help/Optimization_Studio/topics/COS_ home.html

[59] V. Marianov, C. ReVelle, Siting emergency services, In: Z Drezner, (Ed.), Facility Location: A survey of applications and methods, Springer-Verlag, 1995, Ch. 10, pp. 203-227. 